\title{
Cosmology in cubic and $f(P)$ gravity
}

\author{
Cristian Erices, ${ }^{1,2, *}$ Eleftherios Papantonopoulos, ${ }^{1, \dagger}$ and Emmanuel N. Saridakis ${ }^{1,3, \ddagger}$ \\ ${ }^{1}$ Department of Physics, National Technical University of Athens, Zografou Campus GR 157 73, Athens, Greece \\ ${ }^{2}$ Universidad Católica del Maule, Av. San Miguel 3605, Talca, Chile \\ ${ }^{3}$ Department of Astronomy, School of Physical Sciences, \\ University of Science and Technology of China, Hefei 230026, China
}

\begin{abstract}
We construct cubic gravity and its $f(P)$ extension and we investigate their early- and late-time cosmological applications. Cubic gravity is based on a particular invariant $P$, constructed from cubic contractions of the Riemann tensor, under three requirements: (i) the resulting theory possesses a spectrum identical to that of general relativity, (ii) it is neither topological nor trivial in four dimensions, and (iii) it is defined such that it is independent of the dimensions. Relaxing the last condition and restricting the parameters of cubic gravity we can obtain second-order field equations in a cosmological background. We show that at early times one can obtain inflationary, de Sitter solutions, which are driven by an effective cosmological constant constructed purely from the cubic terms of the simple cubic or $f(P)$ gravity. Concerning late-time evolution, the new terms constitute an effective dark-energy sector and we show that the Universe experiences the usual thermal history and the onset of late-time acceleration. In the case of $f(P)$ gravity, depending on the choice of parameters, we find that the dark-energy equation-of-state parameter can be quintessencelike, phantomlike or it can experience the phantom-divide crossing during the evolution, even if an explicit cosmological constant is absent.
\end{abstract}

PACS numbers: 98.80.-k, 95.36.+x, 04.50.Kd

\section{INTRODUCTION}

Higher-order gravities have been introduced in the general framework of modified theories of gravity, with the aim to describe in a uniform way the history of the Universe; to account for the early-time inflation, the late-time acceleration, and the presence of dark matter; and to be consistent with observations [1-5]. A more theoretical motivation in studying higher-order corrections to the Einstein-Hilbert term is that these theories arise naturally in the gravitational effective action of a complete string theory [6] and they result in a renormalizable and thus quantizable gravitational theory [7]. Additionally, in such considerations one can construct theories which possess general relativity as a particular limit [8]. Moreover, certain higher-order gravities are equivalent to Einstein gravity at the linearized level in the vacuum, and the only physical mode propagated by the metric perturbation is a transverse and massless graviton. Such theories are certain f(Lovelock) theories [9].

Higher-order gravities and more generally modified theories of gravity provide a deeper understanding of Einstein gravity theory itself. The construction of modified theories of gravity starts from the Einstein-Hilbert Lagrangian and includes extra terms, such as in $f(R)$ gravity [3, 10-12], $f(G)$ gravity [13, 14], Lovelock gravity [9, 15], Weyl gravity [16, 17], Galileon theory [18-24], etc. More radical modifications of Einstein gravity theory are provided by the introduction of torsion terms in $f(T)$ gravity [25-28] or $f\left(T, T_{G}\right)$ gravity [29,30]. These modified theories of gravity allow us to unveil what features of a gravitational theory are generic and which are specific.

A potential disadvantage of the curvature-based corrections to the Einstein-Hilbert action is that the couplings of the different curvature invariants depend on the spacetime dimension $D$. Hence, they are actually different theories in different dimensions. Furthermore, the higher-order terms may lead to the appearance of higher-than-second-order derivatives in the field equations. Although this does not necessarily mean that the theory presents instabilities and pathologies, since the higher-order derivatives may be just a reflection of healthy extra degrees of freedom (this can be shown through a Hamiltonian analysis), keeping the field equations up to second order is a desirable feature since it does ensure that the theory is pathology free.

One interesting class of modified gravity based on higher-order curvature invariants was recently constructed in [31], and it uses cubic contractions of the Riemann tensor. This theory, called Einsteinian cubic gravity (ECG), possesses basic health conditions coming from nontopological terms. Namely, the coupling parameters, which are dimension

\footnotetext{
*Electronic address: crerices@central.ntua.gr

${ }^{\dagger}$ Electronic address: lpapa@central.ntua.gr

‡Electronic address: msaridak@phys.uoa.gr
} 
independent, allow to propagate a massless and transverse graviton on a maximally symmetric background in the same way as the standard general relativity (GR). In general, such terms contribute with fourth-order derivatives of the metric in the field equations. However, as it was shown in [32-34], the original form of the theory is sufficient to admit spherically symmetric black hole solutions with a second-order differential equation for the metric function. An extra cubic correction, which is trivial for a spherically symmetric black hole ansatz, allows us additionally to accommodate a Friedmann-Lemaître-Robertson-Walker (FLRW) solution with second-order field equations for the scale factor, leading to a purely geometric inflationary period [35] ${ }^{1}$. All these features make this theory physically interesting and hence worthy of further investigation.

In this work we study the cosmological applications of cubic gravity, as well as investigate its modifications, namely $f(P)$ gravity. In particular, we are interested in examining whether the cubic terms $P$ can drive the Universe's acceleration at early and late times, and whether we can obtain the usual thermal history of the Universe. We show that since we are considering four-dimensional spacetimes, the condition of dimension-independent coupling parameter can be relaxed,$^{2}$ allowing us to tune the parameters in order to get second-order field equations for the scale factor.

Inspired by ECG, we obtain a modified theory that shares all the good properties of cubic gravity in four dimensions. In particular, we consider an action with corrections to the Einstein-Hilbert one, constituted by an arbitrary $f(P)$ function of the cubic term. This provides a remarkable advantage over the cosmological models studied in ECG, since it generates inflationary, de Sitter solutions at early times and reproduces the onset of late-time acceleration and the evolution of the densities in agreement with observations, even when the cosmological constant is zero.

The plan of the work is as follows. In Sec. II we construct cubic gravity and we extend it to the $f(P)$ case. Additionally, we apply these theories in a cosmological framework and we extract the Friedmann equations. In Sec. III we perform a detailed investigation of the Universe's behavior at both early and late times, examining various observables such as the effective dark-energy density and equation-of-state parameters. Finally, in Sec. IV are our conclusions.

\section{CUBIC AND $f(P)$ GRAVITY}

In this section we present cubic gravity, as well as its $f(P)$ extension, and we apply them in a cosmological framework. The starting point of cubic gravity is to include additional terms in the Einstein-Hilbert action that are constructed by contractions of three Riemann tensors [31]. Nevertheless, one desires combinations that lead to a theory with the following features: (i) it possesses a spectrum identical to that of general relativity, i.e. the metric perturbation on a maximally symmetric background propagates only a transverse and massless graviton, and (ii) it is neither topological nor trivial in four dimensions. Additionally, we desire that (iii) the theory leads to second-order field equations. In ECG an additional condition is required, namely that the theory should be constructed in a dimension-independent way. However, since in the present work we are interested in the usual four-dimensional cosmological evolution, we relax such a requirement. This freedom allows us to tune the parameters to satisfy condition (iii) without the inclusion of additional cubic terms.

Let us focus on four spacetime dimensions. In this case, a general nontopological [thus condition (iii) is satisfied] cubic term $P$ would be

$$
\begin{aligned}
P= & \beta_{1} R_{\mu}{ }^{\rho}{ }_{\nu}{ }^{\sigma} R_{\rho}{ }^{\gamma}{ }_{\sigma}^{\delta} R_{\gamma}{ }^{\mu}{ }_{\delta}^{\nu}+\beta_{2} R_{\mu \nu}^{\rho \sigma} R_{\rho \sigma}^{\gamma \delta} R_{\gamma \delta}^{\mu \nu}+\beta_{3} R^{\sigma \gamma} R_{\mu \nu \rho \sigma} R^{\mu \nu \rho}{ }_{\gamma}+\beta_{4} R R_{\mu \nu \rho \sigma} R^{\mu \nu \rho \sigma} \\
& +\beta_{5} R_{\mu \nu \rho \sigma} R^{\mu \rho} R^{\nu \sigma}+\beta_{6} R_{\mu}^{\nu} R_{\nu}^{\rho} R_{\rho}^{\mu}+\beta_{7} R_{\mu \nu} R^{\mu \nu} R+\beta_{8} R^{3}
\end{aligned}
$$

where $\beta_{i}$ are parameters. One can show that condition (i) above is satisfied if we impose the parameter relations [31]

$$
\begin{aligned}
& \beta_{7}=\frac{1}{12}\left(3 \beta_{1}-24 \beta_{2}-16 \beta_{3}-48 \beta_{4}-5 \beta_{5}-9 \beta_{6}\right), \\
& \beta_{8}=\frac{1}{72}\left(-6 \beta_{1}+36 \beta_{2}+22 \beta_{3}+64 \beta_{4}+3 \beta_{5}+9 \beta_{6}\right) .
\end{aligned}
$$

One can now use $P$ as a correction term in the Einstein-Hilbert action, and construct the cubic gravity as

$$
S=\int \sqrt{-g} d^{4} x\left[\frac{1}{2 \kappa}(R-2 \Lambda)+\alpha P\right],
$$

\footnotetext{
${ }^{1}$ Black holes solutions were also obtained in [36, 37], alongside the examination of a well-defined cosmology for higher order terms such as the quartic, quintic and infinite tower of higher-curvature corrections to the Einstein-Hilbert action.

2 This can be achieved by considering the dimension-dependent parameters of ECG in [31], namely considering the expressions for the parameters before the dimension-independence condition is imposed, and evaluating in four dimensions.
} 
with $\alpha$ a coupling parameter, $\kappa=8 \pi G$ the Newton's constant, and where we have also allowed for an explicit cosmological constant $\Lambda$ for completeness. We mention here that in principle one should include also quadratic corrections to the above action, such as the Gauss-Bonnet combination $\mathcal{G}=R^{2}-R_{\mu \nu} R^{\mu \nu}+R_{\mu \nu \rho \sigma} R^{\mu \nu \rho \sigma}$. However, in this work we focus on four dimensions and such a term can be neglected since it is topological.

Variation of the action $S+S_{m}$, where $S_{m}$ accounts for the matter Lagrangian $\mathcal{L}_{m}$, leads to the field equations

$$
G_{\mu \nu}+\Lambda g_{\mu \nu}=\kappa\left(T_{\mu \nu}+\alpha H_{\mu \nu}\right)
$$

with

$$
T_{\mu \nu}=-\frac{2}{\sqrt{-g}} \frac{\delta\left(\sqrt{-g} \mathcal{L}_{m}\right)}{\delta g^{\mu \nu}},
$$

representing the matter energy-momentum tensor and $^{3}$

$$
\begin{aligned}
H_{\mu \nu} & =-\frac{2}{\sqrt{-g}} \frac{\delta(\sqrt{-g} P)}{\delta g^{\mu \nu}} \\
& =g_{\mu \nu} P+R^{\alpha \beta \rho}{ }_{(\mu} K_{\nu) \rho \alpha \beta}+2 \nabla^{\alpha} \nabla^{\beta} K_{\alpha(\mu \nu) \beta},
\end{aligned}
$$

where $\nabla_{\mu}$ is the covariant derivative with respect to the spacetime metric $g_{\mu \nu}$. The tensor $K_{\alpha \beta \mu \nu}$ is defined by

$$
\begin{aligned}
K_{\alpha \beta \mu \nu}= & \frac{\partial P}{\partial R^{\alpha \beta \mu \nu}} \\
= & 12\left(\frac{1}{2} R_{\alpha \beta}{ }^{\rho \sigma} R_{\mu \nu \rho \sigma}+6 R_{\alpha}{ }^{\rho}{ }_{[\mu}{ }^{\sigma} R_{\nu] \sigma \beta \rho}+2 g_{\beta[\mu} R_{\nu] \sigma \alpha \rho} R^{\rho \sigma}\right. \\
& \left.-2 g_{\alpha[\mu} R_{\nu] \sigma \beta \rho} R^{\rho \sigma}-4 R_{\rho[\mu} g_{\nu][\alpha} R_{\beta]}{ }^{\rho}-2 R_{\alpha[\mu} R_{\nu] \beta}\right) .
\end{aligned}
$$

The tensor $H_{\mu \nu}$ represents the contribution from the cubic term $P$. Observing the form of $H_{\mu \nu}$ we mention that the complication and length of the above general field equations does not easily allow us to perform the Hamiltonian analysis and check whether they present unhealthy high derivatives, in the sense that they do not correspond to healthy extra degrees of freedom. Nevertheless, this necessary check, i.e. ensuring the condition (iii) above, can be performed around the cosmological background on which we focus in this work, as we will shortly see.

In order to proceed to the cosmological application of cubic gravity we consider a flat homogeneous and isotropic FLRW geometry with metric

$$
d s^{2}=-d t^{2}+a^{2}(t) \delta_{i j} d x^{i} d x^{j}
$$

where $a(t)$ is the scale factor. Additionally, as usual we consider the matter Lagrangian $\mathcal{L}_{m}$ to correspond to a perfect fluid with energy-momentum tensor $T_{\mu \nu}=\left(\rho_{m}+p_{m}\right) u_{\mu} u_{\nu}+p_{m} g_{\mu \nu}$, where $\rho_{m}$ and $p_{m}$ are the energy density and pressure respectively and $u_{\mu}$ is the fluid four-velocity. Under these considerations the general field equations (5) give rise to the Friedmann equations. These equations can now be easily proven to be second order if we make the additional parameter reduction

$$
\beta_{6}=4 \beta_{2}+2 \beta_{3}+8 \beta_{4}+\beta_{5}
$$

In particular, they are written as

$$
\begin{aligned}
3 H^{2} & =\kappa\left(\rho_{m}+6 \alpha \tilde{\beta} H^{6}\right)+\Lambda \\
3 H^{2}+2 \dot{H} & =-\kappa\left[p_{m}-6 \alpha \tilde{\beta} H^{4}\left(H^{2}+2 \dot{H}\right)\right]+\Lambda,
\end{aligned}
$$

where $H=\frac{\dot{a}}{a}$ is the Hubble parameter, with dots denoting derivatives with respect to $t$, and where we have defined the parameter

$$
\tilde{\beta} \equiv-\beta_{1}+4 \beta_{2}+2 \beta_{3}+8 \beta_{4}
$$

\footnotetext{
${ }^{3}$ We use a normalized symmetrization $A_{(\mu \nu)}:=\frac{1}{2}\left(A_{\mu \nu}+A_{\nu \mu}\right)$ and antisymmetrization $A_{[\mu \nu]}:=\frac{1}{2}\left(A_{\mu \nu}-A_{\nu \mu}\right)$.
} 
Finally, note that in FLRW geometry, and under the parameter relations (2) and (10), the cubic invariant $P$ acquires the simple form

$$
P=6 \tilde{\beta} H^{4}\left(2 H^{2}+3 \dot{H}\right)
$$

which includes only up to first-order derivatives and hence the Friedmann equations are up to second order, as indeed they are constructed to be.

Observing the form of the two Friedmann equations we deduce that they can be rewritten in the usual way

$$
\begin{aligned}
3 H^{2} & =\kappa\left(\rho_{m}+\rho_{c u b}\right), \\
3 H^{2}+2 \dot{H} & =-\kappa\left(p_{m}+p_{c u b}\right),
\end{aligned}
$$

where

$$
\begin{aligned}
& \rho_{c u b} \equiv 6 \beta H^{6}+\frac{\Lambda}{\kappa}, \\
& p_{c u b} \equiv-6 \beta H^{4}\left(H^{2}+2 \dot{H}\right)-\frac{\Lambda}{\kappa},
\end{aligned}
$$

and where we have redefined the sole parameter as $\beta \equiv \alpha \tilde{\beta}$. Hence, in cubic gravity we obtain an effective sector that incorporates the effects of the cubic modification of the action. Finally, one can immediately see that this effective sector is conserved, namely

$$
\dot{\rho}_{c u b}+3 H\left(\rho_{c u b}+p_{c u b}\right)=0
$$

while the matter sector is conserved too, namely

$$
\dot{\rho}_{m}+3 H\left(\rho_{m}+p_{m}\right)=0 .
$$

We close this section by constructing $f(P)$ gravity. In the above we described how one can construct a subclass of cubic gravity which in four dimensions is nontopological and in a cosmological spacetime it leads to second-order field equations. The theory is based on the cubic invariant $P$ of (1), under the parameter choices (2) and (10) which ensure the aforementioned requirements. Nevertheless, having such an invariant we may generalize cubic gravity to $f(P)$ gravity, characterized by the action

$$
S=\int \sqrt{-g} d^{4} x\left[\frac{R}{2 \kappa}+f(P)\right],
$$

where $f(P)$ is an arbitrary function of $P$. Variation of the action $S+S_{m}$ leads to the field equations

$$
G_{\mu \nu}=\kappa\left(T_{\mu \nu}+\tilde{H}_{\mu \nu}\right)
$$

with $T_{\mu \nu}$ still given by (6) and

$$
\begin{aligned}
\tilde{H}_{\mu \nu} & =-\frac{2}{\sqrt{-g}} \frac{\delta(\sqrt{-g} f(P))}{\delta g^{\mu \nu}} \\
& =g_{\mu \nu} f(P)+R^{\alpha \beta \rho}\left(\tilde{K}_{\nu) \rho \alpha \beta}+2 \nabla^{\alpha} \nabla^{\beta} \tilde{K}_{\alpha(\mu \nu) \beta} .\right.
\end{aligned}
$$

Here, tensor $\tilde{K}_{\alpha \beta \mu \nu}$ is given in terms of tensor (8) as $\tilde{K}_{\alpha \beta \mu \nu}=f^{\prime}(P) K_{\alpha \beta \mu \nu}$ with primes denoting the derivative of a function with respect to its argument.

In the case of FLRW geometry, the field equations (22) provide the two Friedmann equations, namely

$$
\begin{aligned}
3 H^{2} & =\kappa\left(\rho_{m}+\rho_{f_{P}}\right), \\
3 H^{2}+2 \dot{H} & =-\kappa\left(p_{m}+p_{f_{P}}\right),
\end{aligned}
$$

where

$$
\begin{aligned}
& \rho_{f_{P}} \equiv-f(P)-18 \tilde{\beta} H^{4}\left(H \partial_{t}-H^{2}-\dot{H}\right) f^{\prime}(P), \\
& p_{f_{P}} \equiv f(P)+6 \tilde{\beta} H^{3}\left[H \partial_{t}^{2}+2\left(H^{2}+2 \dot{H}\right) \partial_{t}-3 H^{3}-5 H \dot{H}\right] f^{\prime}(P),
\end{aligned}
$$


and $\partial_{t} \equiv \frac{\partial}{\partial t}$ and $\partial_{t}^{2} \equiv \frac{\partial^{2}}{\partial t^{2}}$. Additionally, note that both the matter and the effective $f(P)$ sector are conserved, namely

$$
\begin{aligned}
\dot{\rho}_{m}+3 H\left(\rho_{m}+p_{m}\right) & =0 \\
\dot{\rho}_{f_{P}}+3 H\left(\rho_{f_{P}}+p_{f_{P}}\right) & =0 .
\end{aligned}
$$

Lastly, in the case where $f(P)=\alpha P-\frac{\Lambda}{\kappa}, f(P)$ gravity gives the simple cubic gravity of (4).

The action (21) can be recast into a (classically) dynamically equivalent action where the system of equations $(24)$ and (25) can be put explicitly as a second-order system by introducing two scalar variables related by $\varphi=f(\phi)$. The details for this procedure can be found in the Appendix.

\section{COSMOLOGICAL APPLICATIONS}

In this section we investigate the cosmological applications of cubic and $f(P)$ gravity at both early and late times. Concerning the early-time application as usual we may neglect the matter sector. We are interested in obtaining the de Sitter solution, which is the basis of the inflation realization. Let us consider first the simple cubic gravity, namely the first Friedmann equation (15) without the matter term. In this case we can easily see that one obtains the de Sitter solution

$$
H^{2}=\frac{6^{1 / 3} \kappa \beta+\zeta^{2 / 3}}{-6^{\frac{2}{3}} \kappa \beta \zeta^{1 / 3}}=\text { const. }
$$

where $\zeta=3 \kappa^{2} \beta^{2} \Lambda+\sqrt{3} \sqrt{\kappa^{3} \beta^{3}\left(3 \kappa \beta \Lambda^{2}-2\right)}$, provided $\beta<0$. When the cosmological constant is absent we get that $H^{2}=(2 \kappa \beta)^{-1 / 2}$ for $\beta>0$. In similar lines, in the case of $f(P)$ gravity, i.e. of the Friedmann equation (24), without the matter sector, we can see that in general we can obtain the de Sitter solution

$$
H^{2}=\frac{\kappa}{3}\left[18 \tilde{\beta} H^{6} f^{\prime}(P)-f(P)\right]=\text { const. }
$$

and $\dot{H}=0$. As we observe, in both theories the de Sitter solution is driven by an effective cosmological constant that is constructed purely from the cubic terms, even if an explicit cosmological constant is absent.

Let us now focus on the late-time evolution. We first examine the basic cubic gravity, namely (4), where the correction in the general relativity action is just $P$. In this case the cubic correction constitutes the effective darkenergy sector, whose energy density and pressure are

$$
\begin{aligned}
& \rho_{D E} \equiv \rho_{c u b} \\
& p_{D E} \equiv p_{c u b},
\end{aligned}
$$

with $\rho_{c u b}$ and $p_{c u b}$ given respectively by (17) and (18), while the effective dark-energy equation-of-state parameter is

$$
w_{D E} \equiv \frac{p_{D E}}{\rho_{D E}} .
$$

In order to proceed we define the density parameters $\Omega_{i} \equiv \kappa \rho_{i} /\left(3 H^{2}\right)$, with $i$ standing for matter and dark energy. Lastly, it proves convenient to introduce the deceleration parameter $q$, which reads as

$$
q \equiv-1-\frac{\dot{H}}{H^{2}}=\frac{1}{2}+\frac{3}{2}\left(w_{m} \Omega_{m}+w_{D E} \Omega_{D E}\right) .
$$

We elaborate the Friedmann equations (15) and (16) numerically, using the redshift $z$ as the independent variable, defined as $1+z=a_{0} / a$ with the present scale factor set to $a_{0}=1$. Concerning the initial conditions, we set $\Omega_{D E}(z=0) \equiv \Omega_{D E 0} \approx 0.69$ and thus $\Omega_{m}(z=0) \equiv \Omega_{m 0} \approx 0.31$ as required by observations [38]. Additionally, we consider the matter sector to be dust, namely we set $w_{m}=0$. In the upper graph of Fig. 1 we depict $\Omega_{D E}(z)$ and $\Omega_{m}(z)=1-\Omega_{D E}(z)$. In the middle graph we present the corresponding behavior of $w_{D E}(z)$ given by $(34)$. Finally, in the lower graph we show the deceleration parameter as it arises from (35).

From the upper graph of Fig. 1 we observe that we can obtain the usual thermal history, that is the sequence of matter and dark-energy eras, while in the future $(z \rightarrow-1)$ the Universe asymptotically results in a complete dark-energy dominated, de Sitter phase. Moreover, from the third graph of Fig. 1 we see that the transition from deceleration to acceleration is realized at $z \approx 0.5$ in agreement with the observed behavior. Additionally, from the 

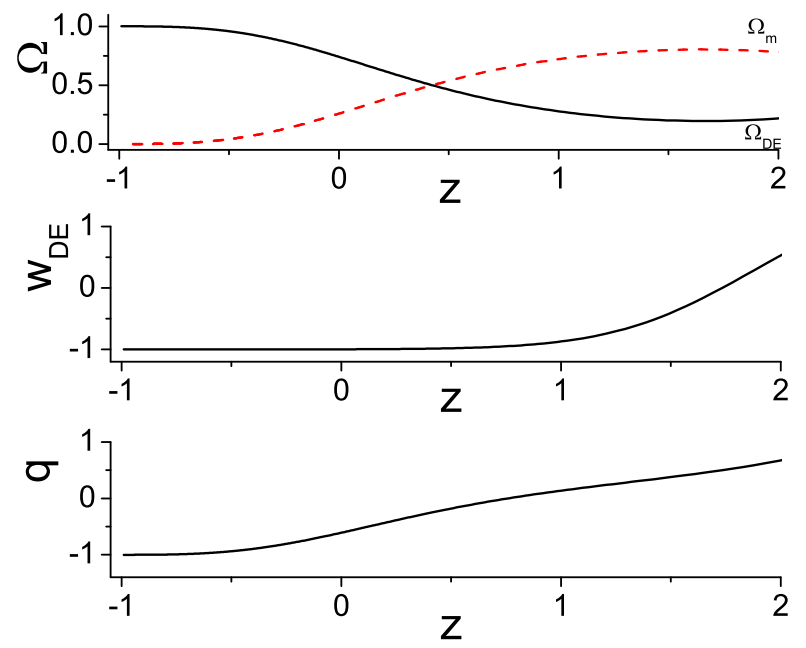

FIG. 1: Upper graph: The evolution of the effective dark energy density parameter $\Omega_{D E}$ (black-solid) and of the matter density parameter $\Omega_{m}$ (red-dashed), as a function of the redshift $z$, in the case of simple cubic gravity (4), for $\beta=0.005$ and $\Lambda=1$, in units where $\kappa=1$. We have imposed the initial conditions $\Omega_{D E}(z=0) \equiv \Omega_{D E 0} \approx 0.69$ in agreement with observations. Middle graph: The evolution of the corresponding effective dark energy equation-of-state parameter $w_{D E}$ from (34). Lower graph: The evolution of the corresponding deceleration parameter $q$ from (35).

middle graph of Fig. 1 we observe that the effective dark-energy equation-of-state parameter $w_{D E}$ quickly acquires the value -1 .

Nevertheless, we must mention here that in the simple cubic gravity (4) the late-time acceleration is triggered mainly by the explicit cosmological constant term $\Lambda$, while the cubic term, quantified by the parameter $\beta$ in (17) and (18), plays a secondary role (a similar result was found in a different context in [35]). The reason is that since the cubic correction in (17) is proportional to $H^{6}$, if this term is to play the main role in the late-time acceleration then at earlier times it will be large enough in order to spoil the desired thermal history of the Universe and lead to an "early dark energy" [39] behavior. However, even in the case where the cubic term plays a secondary role in the late-time acceleration of the Universe, its effect can still be significant at intermediate times (see the middle graph of Fig. 1 where the deviation from $\Lambda$ CDM scenario is clear) as well as at the perturbation level reflected in the matter clustering and the large scale structure (LSS). The detailed analysis of the perturbations and the comparison with LSS data such as the $f \sigma_{8}$ ones will be handled in a separate project.

Let us now investigate the extended cubic gravity, namely the $f(P)$ gravity (21). In this case, using (26) and (27), the effective dark-energy density and pressure are given by

$$
\begin{aligned}
& \rho_{D E} \equiv \rho_{f_{P}} \\
& p_{D E} \equiv p_{f_{P}},
\end{aligned}
$$

and the effective dark-energy equation-of-state parameter as usual is $w_{D E} \equiv p_{D E} / \rho_{D E}$. As a first example we consider the case where

$$
f(P)=\alpha \sqrt{P}
$$

without considering an explicit cosmological constant. Additionally, we merger the coupling parameter $\alpha$ with the parameter $\tilde{\beta}$ of $P$ in (14) through $\beta \equiv \alpha \tilde{\beta}$.

We elaborate the Friedmann equations (24) and (25) numerically, considering the matter sector to be dust. In the upper graph of Fig. 2 we present $\Omega_{D E}(z)$ and $\Omega_{m}(z)$, in the middle graph we draw $w_{D E}(z)$, and in the lower graph we depict the deceleration parameter $q(z)$. As we observe, once again we obtain the sequence of matter and dark-energy eras and the onset of late-time acceleration. However, the significant advantage compared to the simple cubic gravity of Fig. 1 is that now the above behavior is obtained although we have not considered an explicit cosmological constant; namely it arises purely from the $f(P)$ modification. This can also be seen in the evolution of $w_{D E}$, which exhibits a dynamical behavior different than $\Lambda \mathrm{CDM}$ at all times, with an asymptotic value slightly into the phantom regime. 

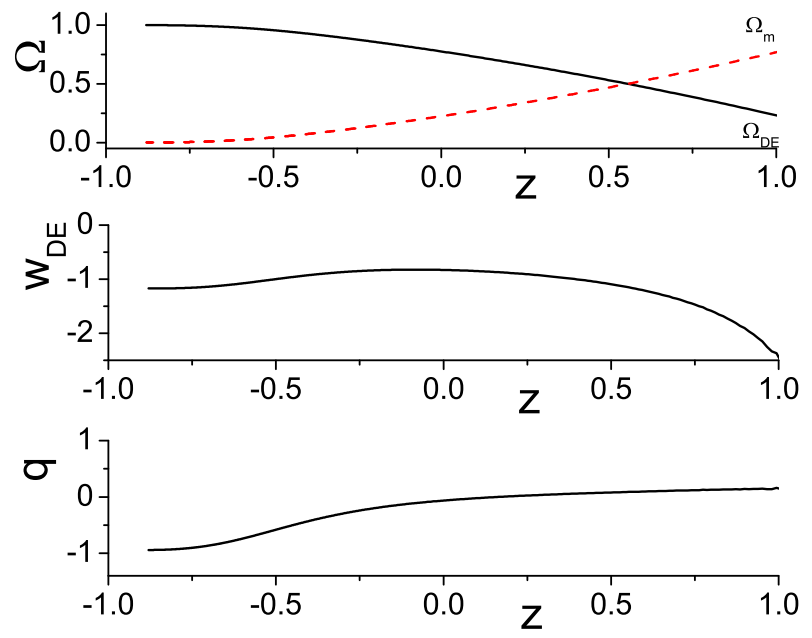

FIG. 2: Upper graph: The evolution of the effective dark-energy density parameter $\Omega_{D E}$ (black-solid) and of the matter density parameter $\Omega_{m}$ (red-dashed), as a function of the redshift $z$, in the case of $f(P)$ gravity (21), under the square choice (38), for $\beta=5$ in units where $\kappa=1$. We have imposed the initial conditions $\Omega_{D E}(z=0) \equiv \Omega_{D E 0} \approx 0.69$ in agreement with observations. Middle graph: The evolution of the corresponding effective dark energy equation-of-state parameter $w_{D E}$. Lower graph: The evolution of the corresponding deceleration parameter $q$.

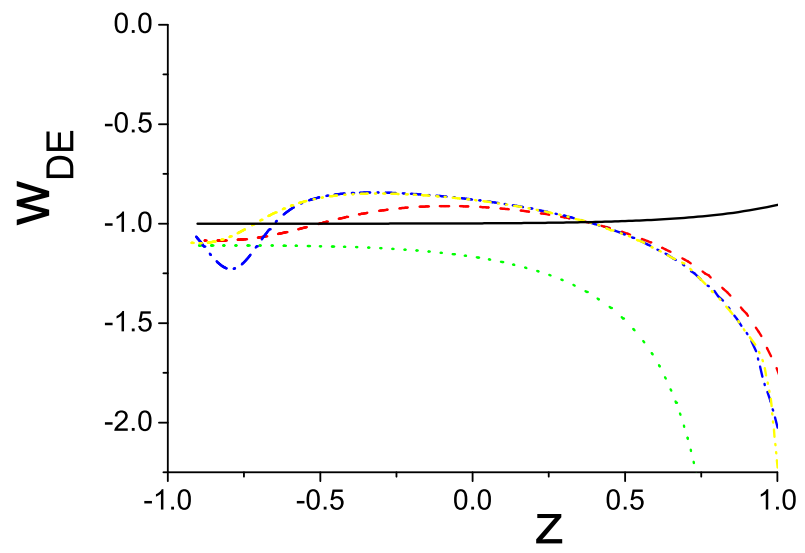

FIG. 3: The evolution of the equation-of-state parameter $w_{D E}$ of the effective dark energy of $f(P)$ gravity, for various forms of $f(P): f(P)=\beta P-\frac{\Lambda}{\kappa}$ with $\beta \equiv \alpha \tilde{\beta}=0.005$ and $\Lambda=1$ (black - solid), $f(P)=\alpha \sqrt{P}$ with $\beta \equiv \alpha \tilde{\beta}=5$ (red - dashed), $f(P)=\alpha P^{1 / 3}$ with $\beta \equiv \alpha \tilde{\beta}=-10$ (green - dashed), $f(P)=\alpha \sqrt{P}+\gamma P$ with $\beta \equiv \alpha \tilde{\beta}=1$ and $\zeta \equiv \gamma \tilde{\beta}=0.1$ (blue dashed-dotted), and $f(P)=\alpha P^{1 / 3}+\gamma P$ with $\beta \equiv \alpha \tilde{\beta}=5$ and $\zeta \equiv \gamma \tilde{\beta}=0.050$ (yellow dashed-dotted). We have imposed the initial conditions $\Omega_{D E}(z=0) \equiv \Omega_{D E 0} \approx 0.69$ and we use units where $\kappa=1$.

In order to examine the effect of $f(P)$ forms on $w_{D E}$, in Fig. 3 we present the evolution of $w_{D E}(z)$ for various $f(P)$ choices. In particular, apart from the simple cubic gravity of Fig. 1, which is obtained for $f(P)=\beta P-\frac{\Lambda}{\kappa}$, and for the square-root $f(P)$ gravity of (38) of Fig. 2, we consider the cubic-root case $f(P)=\alpha P^{1 / 3}$, as well as the combined cases $f(P)=\alpha \sqrt{P}+\gamma P$ and $f(P)=\alpha P^{1 / 3}+\gamma P$.

As we observe, one can fix the model parameters in order to obtain a $w_{D E}$ at present $(z=0)$ close to the cosmological constant value, in agreement with observations, while the past and future behavior can be different for different scenarios. As we mentioned earlier, we note that apart from the linear case, such behavior is achieved without the need for an explicit cosmological constant, which is a significant advantage. Furthermore, note that $w_{D E}$ can be 
lying in the quintessence regime or in the phantom regime or it can experience the phantom-divide crossing during the cosmological evolution, which reveals the capabilities of the theory at hand, since it is known that the phantom regime cannot be easily obtained [40].

\section{CONCLUSIONS}

In this work we constructed cubic gravity and its extension and we investigated their cosmological applications for both the early- and late-time Universe. Cubic gravity is based on the invariant $P$ that is constructed using particular cubic contractions of the Riemann tensor, such that the theory (i) possesses a spectrum identical to that of Einstein gravity, i.e. the metric perturbation on a maximally symmetric background propagate only a transverse and massless graviton; (ii) it is neither topological nor trivial in four dimensions, and (iii) it is defined such that it is independent of the dimensions. By relaxing this last condition, and tuning the coupling parameters, the theory admits second-order field equations for a four-dimensional cosmological ansatz without the inclusion of any additional term. These features make cubic gravity a well-behaved candidate for modified gravity. Additionally, apart from simple cubic gravity, which uses $P$ in the Lagrangian, we constructed extensions, namely $f(P)$ gravity.

Applying both simple cubic and $f(P)$ gravity in a cosmological framework, we saw that at early times one can easily obtain inflationary, de Sitter solutions, which are driven by an effective cosmological constant that is constructed purely from the cubic terms, even if an explicit cosmological constant is absent.

Concerning the late-time evolution, we showed that the cubic terms construct an effective dark-energy sector of gravitational origin. In the case of simple cubic gravity we obtained the usual thermal history, i.e. the sequence of matter and dark-energy eras, with the onset of late-time acceleration at around $z \approx 0.5$ in agreement with observations, while in the future the Universe asymptotically results in a completely dark-energy dominated, de Sitter phase. Nevertheless, if we desire not to spoil the Universe's evolution at early times then we find that the background latetime acceleration is triggered mainly by the explicit cosmological constant, with the cubic term playing a secondary role (which could however be significant at the perturbation level).

However, when we proceeded to $f(P)$ gravity we found that we can obtain the usual thermal history and the onset of late-time acceleration even without considering an explicit cosmological constant, namely as a result of the $f(P)$ corrections purely. Moreover, examining the evolution of the effective dark-energy equation-of-state parameter $w_{D E}$ we showed that its behavior is determined by the $f(P)$ form as well as the parameter choices, and it can be quintessencelike or phantomlike or it can experience the phantom-divide crossing during the evolution. Thlikeese features reveal the capabilities of $f(P)$ gravity and offer a good motivation for its further investigation.

Finally, we mention that there are additional studies that need to be performed before cubic and $f(P)$ gravity can be considered as a successful candidate for the description of nature. Firstly, one should use data from type Ia supernovae (SNIa), cosmic microwave background (CMB) shift parameter, baryon acoustic oscillations (BAO), and direct Hubble constant observations, in order to extract constraints on the involved forms and parameters. Furthermore, one needs to perform a detailed perturbation analysis and confront the theory with data from CMB temperature and polarization as well as from LSS (such as the $\sigma_{8}$ ones). Moreover, one could perform a phase-space analysis in order to reveal the global behavior of $f(P)$ cosmology. Lastly, going beyond the cosmological framework, as we mentioned above the original ECG theory can be corrected in order to admit black hole and cosmological solutions; however these solutions coexist in a disjoint set of values for the cubic coupling parameter. It would be interesting to investigate whether the above $f(P)$ modification provides enough freedom to obtain black hole solutions in a common range of parameters. These necessary investigations lie beyond the scope of this work and are left for future projects.

\section{Acknowledgments}

This article is based upon work from COST Action "Cosmology and Astrophysics Network for Theoretical Advances and Training Actions," supported by the European Cooperation in Science and Technology (COST). C.E. acknowledges financial support given by Becas Chile, Comisión Nacional de Investigación Científica y Tecnológica. 


\section{Appendix: Equations of motion in $f(P)$ gravity as second-order field equations}

The $f(P)$ gravity can be recast into a (classically) dynamically equivalent action where the Friedmann equations (24) and (25) can be put explicitly as a second-order system. For this let us consider the following action endowed with scalar variables $\varphi$ and $\phi$ :

$$
S=\int \sqrt{-g} d^{4} x\left[\frac{R}{2 \kappa}+f(\phi)+\varphi(P-\phi)\right]
$$

Variation with respect to $\varphi$ yields $\phi=P$. Replacing this algebraic constraint back into action (A.1) yields the action (21). Considering the variation with respect to $\phi$, it is obtained that $\varphi=f^{\prime}(\phi)$. Variation with respect to the metric $g_{\mu \nu}$ leads to

$$
\begin{aligned}
G_{\mu \nu} & =\kappa\left(T_{\mu \nu}+\tilde{H}_{\mu \nu}\right) \\
\tilde{H}_{\mu \nu} & \equiv g_{\mu \nu} f(\phi)+\varphi R^{\alpha \beta \rho}{ }_{(\mu} K_{\nu) \rho \alpha \beta}+2 \nabla^{\alpha} \nabla^{\beta}\left[\varphi K_{\alpha(\mu \nu) \beta}\right],
\end{aligned}
$$

which retrieves equations equivalent to (23) when the algebraic constraints are considered. Then, the Friedmann equations (24) and (25), take the same form but with an effective sector given by

$$
\begin{aligned}
& \rho_{f_{P}} \equiv-f(\phi)-18 \tilde{\beta} H^{4}\left(H \partial_{t}-H^{2}-\dot{H}\right) \varphi \\
& p_{f_{P}} \equiv f(\phi)+6 \tilde{\beta} H^{3}\left[H \partial_{t}^{2}+2\left(H^{2}+2 \dot{H}\right) \partial_{t}-3 H^{3}-5 H \dot{H}\right] \varphi,
\end{aligned}
$$

which clearly possesses second-order field equations.

[1] S. Nojiri and S. D. Odintsov, "Introduction to modified gravity and gravitational alternative for dark energy," Int. J. Geom. Meth. Mod. Phys. 04, 115 (2007). [hep-th/0601213].

[2] T. Clifton, P. G. Ferreira, A. Padilla and C. Skordis, "Modified Gravity and Cosmology," Phys. Rept. 513, 1 (2012) [arXiv:1106.2476 [astro-ph.CO]].

[3] S. Nojiri and S. D. Odintsov, "Unified cosmic history in modified gravity: from F(R) theory to Lorentz non-invariant models," Phys. Rept. 505, 59 (2011) [arXiv:1011.0544 [gr-qc]].

[4] E. J. Copeland, M. Sami and S. Tsujikawa, "Dynamics of dark energy," Int. J. Mod. Phys. D 15, 1753 (2006)

[5] Y. F. Cai, S. Capozziello, M. De Laurentis and E. N. Saridakis, "f(T) teleparallel gravity and cosmology," Rept. Prog. Phys. 79, no. 10, 106901 (2016) [arXiv:1511.07586 [gr-qc]].

[6] D. J. Gross and J. H. Sloan, "The Quartic Effective Action for the Heterotic String," Nucl. Phys. B 291, 41 (1987).

[7] K. S. Stelle, "Renormalization of Higher Derivative Quantum Gravity," Phys. Rev. D 16, 953 (1977).

[8] T. Biswas, E. Gerwick, T. Koivisto and A. Mazumdar, "Towards singularity and ghost free theories of gravity," Phys. Rev. Lett. 108, 031101 (2012). arXiv:1110.5249.

[9] D. Lovelock, "The Einstein tensor and its generalizations," J. Math. Phys. 12, 498 (1971).

[10] A. A. Starobinsky, "A New Type of Isotropic Cosmological Models Without Singularity," Phys. Lett. B 91, 99 (1980).

[11] A. De Felice and S. Tsujikawa, "f(R) theories," Living Rev. Rel. 13, 3 (2010), [arXiv:1002.4928 [gr-qc]].

[12] S. Capozziello, "Curvature quintessence," Int. J. Mod. Phys. D 11, 483 (2002), arXiv:gr-qc/0201033.

[13] S. Nojiri and S. D. Odintsov, "Modified Gauss-Bonnet theory as gravitational alternative for dark energy," Phys. Lett. B 631, 1 (2005), arXiv:hep-th/0508049.

[14] A. De Felice and S. Tsujikawa, "Construction of cosmologically viable $\mathrm{f}(\mathrm{G})$ dark energy models," Phys. Lett. B 675, 1 (2009), arXiv:0810.5712.

[15] N. Deruelle and L. Farina-Busto, "The Lovelock Gravitational Field Equations in Cosmology," Phys. Rev. D 41, 3696 (1990).

[16] P. D. Mannheim and D. Kazanas, "Exact Vacuum Solution to Conformal Weyl Gravity and Galactic Rotation Curves," Astrophys. J. 342, 635 (1989).

[17] E. E. Flanagan, "Fourth order Weyl gravity," Phys. Rev. D 74, 023002 (2006), arXiv:astro-ph/0605504.

[18] A. Nicolis, R. Rattazzi and E. Trincherini, "The Galileon as a local modification of gravity," Phys. Rev. D 79, 064036 (2009), arXiv:0811.2197.

[19] C. Deffayet, G. Esposito-Farese and A. Vikman, "Covariant Galileon," Phys. Rev. D 79, 084003 (2009), arXiv:0901.1314.

$[20]$ G. Leon and E. N. Saridakis, "Dynamical analysis of generalized Galileon cosmology," JCAP 1303, 025 (2013), arXiv:1211.3088.

[21] T. Kolyvaris, G. Koutsoumbas, E. Papantonopoulos and G. Siopsis, "Scalar Hair from a Derivative Coupling of a Scalar Field to the Einstein Tensor," Class. Quant. Grav. 29, 205011 (2012) [arXiv:1111.0263 [gr-qc]]. 
[22] A. Cisterna and C. Erices, "Asymptotically locally AdS and flat black holes in the presence of an electric field in the Horndeski scenario," Phys. Rev. D 89, 084038 (2014) [arXiv:1401.4479 [gr-qc]].

[23] C. Erices and C. Martínez, "Stationary cylindrically symmetric spacetimes with a massless scalar field and a nonpositive cosmological constant," Phys. Rev. D 92, no. 4, 044051 (2015) [arXiv:1504.06321 [gr-qc]].

[24] C. Erices and C. Martínez, "Rotating hairy black holes in arbitrary dimensions," Phys. Rev. D 97, no. 2, 024034 (2018) [arXiv:1707.03483 [hep-th]].

[25] G. R. Bengochea and R. Ferraro, "Dark torsion as the cosmic speed-up," Phys. Rev. D 79, 124019 (2009), arXiv:0812.1205.

[26] E. V. Linder, "Einstein's Other Gravity and the Acceleration of the Universe," Phys. Rev. D 81, 127301 (2010), arXiv:1005.3039.

[27] S. H. Chen, J. B. Dent, S. Dutta and E. N. Saridakis, "Cosmological perturbations in f(T) gravity," Phys. Rev. D 83, 023508 (2011), arXiv:1008.1250.

[28] G. Kofinas, E. Papantonopoulos and E. N. Saridakis, "Self-Gravitating Spherically Symmetric Solutions in Scalar-Torsion Theories," Phys. Rev. D 91, no. 10, 104034 (2015) [arXiv:1501.00365 [gr-qc]].

[29] G. Kofinas and E. N. Saridakis, "Teleparallel equivalent of Gauss-Bonnet gravity and its modifications," Phys. Rev. D 90, 084044 (2014), arXiv:1404.2249.

[30] G. Kofinas and E. N. Saridakis, "Cosmological applications of $F\left(T, T_{G}\right)$ gravity," Phys. Rev. D 90, 084045 (2014), arXiv:1408.0107.

[31] P. Bueno and P. A. Cano, "Einsteinian cubic gravity," Phys. Rev. D 94, no. 10, 104005 (2016) [arXiv:1607.06463 [hep-th]].

[32] P. Bueno and P. A. Cano, "Four-dimensional black holes in Einsteinian cubic gravity," Phys. Rev. D 94, no. 12, 124051 (2016) [arXiv:1610.08019 [hep-th]].

[33] R. A. Hennigar and R. B. Mann, "Black holes in Einsteinian cubic gravity," Phys. Rev. D 95, no. 6, 064055 (2017) [arXiv:1610.06675 [hep-th]].

[34] R. A. Hennigar, D. Kubizňák and R. B. Mann, "Generalized quasitopological gravity," Phys. Rev. D 95, no. 10, 104042 (2017) [arXiv:1703.01631 [hep-th]].

[35] G. Arciniega, J. D. Edelstein and L. G. Jaime, "Towards purely geometric inflation and late time acceleration," arXiv:1810.08166 [gr-qc].

[36] G. Arciniega, P. Bueno, P. A. Cano, J. D. Edelstein, R. A. Hennigar and L. G. Jaime, "Geometric Inflation," arXiv:1812.11187 [hep-th].

[37] A. Cisterna, N. Grandi and J. Oliva, "On four-dimensional Einsteinian gravity, quasitopological gravity, cosmology and black holes," arXiv:1811.06523 [hep-th].

[38] P. A. R. Ade et al. [Planck Collaboration], "Planck 2015 results. XIII. Cosmological parameters," Astron. Astrophys. 594, A13 (2016) [arXiv:1502.01589 [astro-ph.CO]].

[39] M. Bartelmann, M. Doran and C. Wetterich, "Non-linear structure formation in cosmologies with early dark energy," Astron. Astrophys. 454, 27 (2006) [astro-ph/0507257].

[40] S. Nojiri and E. N. Saridakis, "Phantom without ghost," Astrophys. Space Sci. 347, 221 (2013) [arXiv:1301.2686 [hep-th]]. 